\title{
X Congreso Ilbérico de Agroingeniería

\section{Análisis resistente de viales de jabre estabilizados con los cementos: CEM II B-L 32,5N y CEM II A-V 42.5N}

\author{
C. Gilarranz ${ }^{1}$, S. Laserna ${ }^{2}$, J. Montero \\ 1 Universidad Politécnica de Madrid; carlosandres.gilarranz@upm.es \\ 2 Autónomo; santiago.laserna@agroes.es \\ 3 Universidad de Castilla-La Mancha; jesus.montero@uclm.es
}

\begin{abstract}
Resumen: En el presente trabajo se pretende hacer una comparativa de las propiedades resistentes de un mismo suelo de jabre estabilizado con 2 tipos de cemento; CEM II B-L 32,5N y CEM II A-V 42.5N, analizando la influencia de la dosificación del cemento y el tiempo de curado sobre la resistencia del suelo a compresión, y establecer unas recomendaciones técnicas de su utilización que cumplan las indicaciones del Pliego de Prescripciones Técnicas Generales para Obras de Carreteras y Puentes. Esto se realizará analizando la influencia de la dosificación del cemento (de 0 a 10\%) y el tiempo de curado (2, 7 y 21 días) sobre la resistencia del suelo a compresión, así como comparar resultados con los estudios previos realizados con ambos tipos de cemento.
\end{abstract}

Palabras clave: caminos, geotecnia, mecánica de suelos

\section{Introducción}

El jabre se presenta en yacimientos sedimentarios como depósito de fragmentos de una roca granítica que se ha desagregado por la acción de los agentes atmosféricos (meteorización) y descomposición, que han permanecido in situ o muy próximos a la roca madre. Posee un ángulo de rozamiento en torno a $28-32^{\circ}$.

Dependiendo de ese nivel de descomposición, desde el punto de vista granulométrico, este material se puede agrupar en tres categorías: jabre de arena gruesa, jabre de arena fina y jabre arcilloso.

La principal característica debida a su composición granítica es la ausencia de barro una vez mojado. Esto lo convierte en un material que tiene muchas ventajas en comparación con otros, pues tiene bajo coste, alta rapidez en la ejecución y bajo coste de mantenimiento. Es un material que tradicionalmente ya se utilizaba en tiempos de los romanos en sus calzadas [1] y que desde pocos años a acá se ha reimpulsado su uso, como en la rehabilitación de caminos del Parque del Oeste en Madrid o en varios caminos en la urbanización La Moraleja en Madrid[2].

\section{Materiales}

\section{Cemento, jabre y agua}

Los materiales utilizados en la presente experimentación fueron cemento, jabre y agua.

El cemento es un conglomerante hidráulico, es decir, un material inorgánico finamente molido que amasado con agua, forma una pasta que fragua y endurece por medio de reacciones y procesos de hidratación y que, una vez endurecido conserva su resistencia y estabilidad incluso bajo el agua. Dosificado y mezclado apropiadamente con agua y áridos produce un hormigón o 


\section{CONGRESO IBÉRICO DE AGROINGENIERÍA \\ X CONGRESSO IBÉRICO DE AGROENGENHARIA \\ 3 - 6 septiembre 2019, Huesca - España}

mortero que conserva su trabajabilidad durante un tiempo suficiente alcanzando unos niveles de resistencias preestablecido y presentando una estabilidad a largo plazo.

Uno de los cementos Portland es el CEM II B-L 32.5N, con la denominación Cemento portland con caliza, y con los siguientes componentes[3]: Clínker (65-79\%), Caliza (21-35\%) y componentes minoritarios (0-5\%). La elección de este tipo de cemento se hizo por llevar un alto porcentaje de caliza ya que ésta participa activamente en el proceso de la hidratación, favoreciendo este proceso, así como también hacer de filler calizo.

El otro cemento Portland es el CEM II A-V 42.5N, con la denominación Cemento portland con cenizas volantes, y con los siguientes componentes[3]: Clínker $85 \%$, Cenizas volantes $10 \%$ y componentes minoritarios $5 \%$ que en su mayoría son reguladores del fraguado como es el yeso $3 \%$. La elección de este tipo de cemento se hizo por llevar un alto porcentaje de cenizas volantes ya que éstas mejoran la trabajabilidad y proporcionan menor calor de hidratación lo que hace que haya una menor retracción y fisuración al disminuir la velocidad global de hidratación y por lo tanto enlentecer el fraguado, siendo los cementos puzolánicos recomendados para firmes de carreteras, según enunciaba ya [4].

El agua a emplear, tanto para el amasado como para el curado del hormigón en obra, no debe contener ningún ingrediente en cantidades tales que afecte negativamente a las propiedades del mortero u hormigón. Considerándose que las aguas potables son aptas para el uso en adición con cemento.

Así el agua debe cumplir los siguientes requisitos mínimos:

- Exponente de hidrógeno. $\mathrm{PH}>5$.

- Sustancias disueltas < 15 gramos por litro (15.000 p.p.m.).

- Sulfatos, expresados en SO4, máximo de 1 gramo por litro (1.000 p.pm.).

- Hidratos de carbono, deben ausentarse.

- Sustancias orgánicas disueltas en éter < de 15 gramos por litro (15.000 p.p.m.).

En este ensayo se ha utilizado agua corriente proporcionada por el Canal de Isabel II, que proviene en mayoría de los embalses localizados en la zona norte de la Comunidad de Madrid. Éste agua es utilizada habitualmente como agua potable, por lo que cumple con las necesidades expuestas en el PG-3.

La composición mineralógica del jabre está formada por arenas de cuarzos granulares (pseudoesféricos), feldespatos (ortosa), turmalinas, biotitas, etc., unidas por partículas arcillosas (caolinita). Se encuentra en las zonas meteorizadas de los grandes batolitos graníticos próximos a la calzada romana, de donde se extrae con facilidad mediante medios mecánicos rudimentarios dada su escaso grado de compactación[5].

Ya por úlimo, este material, el jabre, según el artículo 513 del Pliego de prescripciones técnicas generales para obras de carreteras y puentes, cumple con las características previas que deberían cumplir los suelos para que al estabilizarlos con cemento resultase un material con características técnicas adecuadas y económicamente admisible[6].

\section{Probetas y aparataje}

Las probetas se realizan con un molde cilíndrico de metal de $102 \mathrm{~mm}+/-0,4 \mathrm{~mm}$ de diámetro interior y $122,4 \mathrm{~mm}+/-0,1 \mathrm{~mm}$ de altura, equivalente a un volumen de $1000 \mathrm{~cm}^{3}$ y debe disponer de un collar con el mismo diámetro y una altura aproximada de $60 \mathrm{~mm}$, para colocarlo en la parte superior del molde en el momento del apisonado.
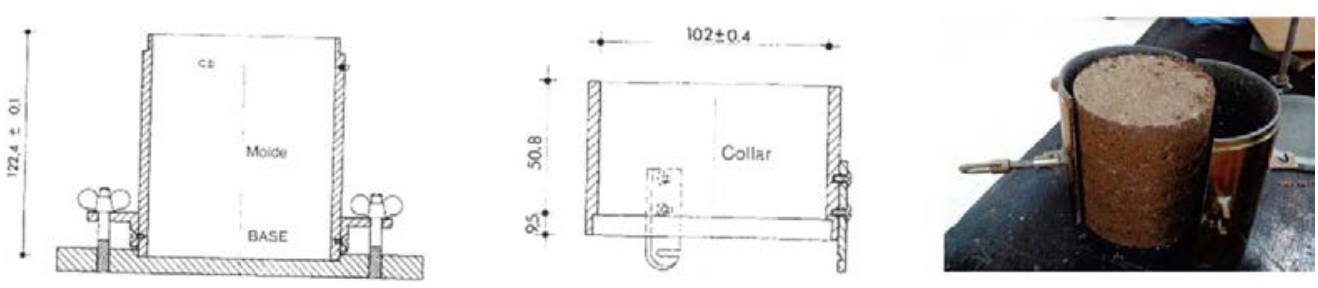

Figura 1. Molde y probeta 


\section{CONGRESO IBÉRICO DE AGROINGENIERÍA \\ X CONGRESSO IBÉRICO DE AGROENGENHARIA \\ 3 - 6 septiembre 2019, Huesca - España}

Para la fabricación de estas probetas y poder realizarse el ensayo Proctor Normal fue necesaria la compactadora que tiene una maza metálica de $2,5 \mathrm{~kg}+/-0,01 \mathrm{~kg}$, adaptada al interior de una guía tubular adecuada para que a altura de caída libre sea de $305 \mathrm{~mm}+/-2 \mathrm{~mm}$. La maza puede ser manual, aunque se utilizó compactadora automática que distribuye los golpes de manera uniforme sobre la superficie del material. El procedimiento de fabricación de esta era el de llenar el molde con el collar superior colocado mediante la porción de jabre-cemento mezclada con agua, repartida en tres capas aproximadamente iguales, de forma que cada capa después de ser compactada, quedara con una altura superior a un tercio de la altura del molde. La compactación de cada una de estas capas se realiza por medio de 26 golpes de maza distribuidos uniformemente. Con este procedimiento se normaliza la energía de compactación de $0,583 \mathrm{~J} / \mathrm{cm}^{3}$ que se le ha imprimido a cada probeta.
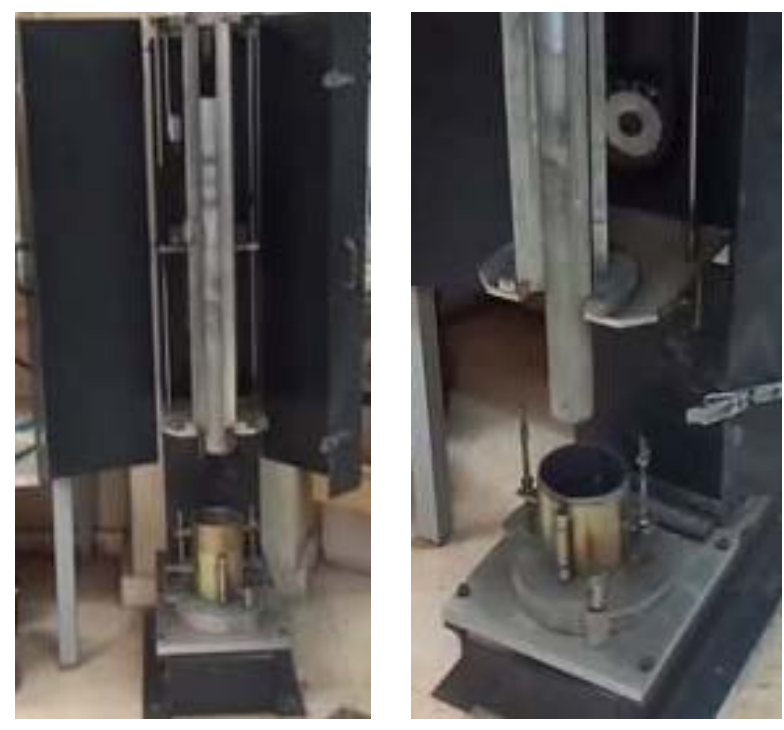

Figura 2. Compactadora

Para la rotura de estas probetas se utilizó la prensa multiensayo. La probeta se coloca entre los cabezales de la prensa, se acciona el sistema y a continuación el dispositivo avanza lo estrictamente necesario para que el cabezal y la probeta entren en contacto. Una vez conseguido este acoplamiento se inicia el proceso de carga de la probeta de tal forma que el tiempo final de rotura esté comprendido entre 1 y 10 minutos. Una vez conseguido esto, se observan las cargas y deformaciones cada 30 segundos hasta que el valor de la carga comience a disminuir, dicho valor es anotado ya que coincide con la carga máxima soportable de la probeta o lo que se conoce como carga de rotura.
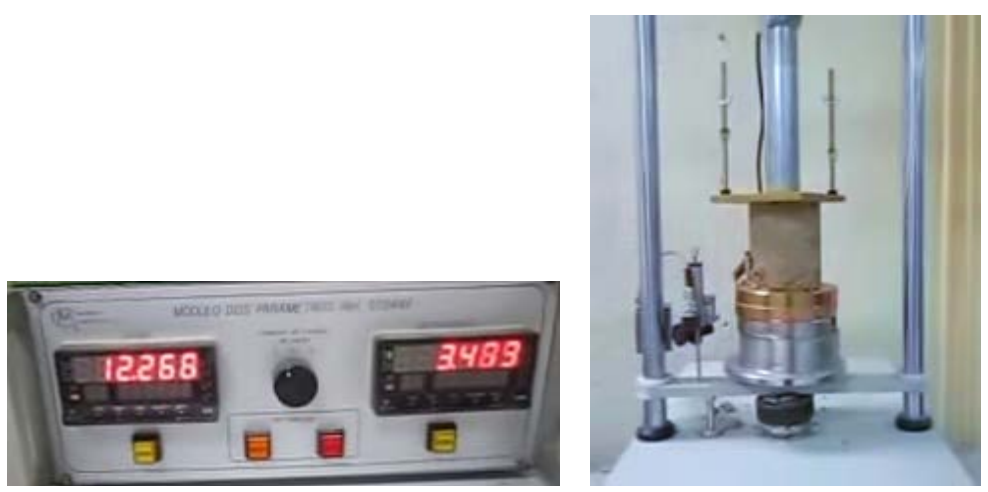

Figura 3. Rotura en prensa multiensayo 


\section{CONGRESO IBÉRICO DE AGROINGENIERÍA \\ X CONGRESSO IBÉRICO DE AGROENGENHARIA \\ 3 - 6 septiembre 2019, Huesca - España}

\section{Métodos}

La metodología empleada ha sido la Norma UNE para los ensayos, tanto al jabre como a la mezcla.

3.1. Metodología de ensayos realizados al jabre.

o Granulometría por tamizado, según norma [7].

o Determinación del Límite Pástico [8].

o Determinación del Límite Líquido [9].

o Ensayo de Compactación. Proctor Normal [10].

Siguiendo esta metodología se ha pretendido caracterizar este material base de este tipo de estabilizaciones, justificando su idoneidad para ser estabilizado con cemento.

\subsection{Metodología de ensayos realizados a la mezcla jabre-cemento}

- Mezclas de áridos sin ligante y con conglomerante hidráulico. Parte 41: método de ensayo para la determinación de la resistencia a la compresión de las mezclas de áridos con conglomerante hidráulico [11].

Una vez determinada la curva humedad-densidad, se determinó la humedad óptima que proporcionaba la máxima densidad y ya con este valor se determinaron tres ensayos bien diferenciados, correspondiendo estos a los días de curado de las probetas ( 2,7 y 21 días) a la mezcla de jabre estabilizado con cemento. Se analizó también como variable el contenido en cemento (\%CEM), del 0-1,5-2,5-3,5-4,5-5,5-6,5-7,5-8,5-10\%, con tres repeticiones por muestra y poder así tratar estadísticamente los datos obtenidos por medio de un análisis de la varianza ANOVA.

Tanto para el jabre como para la mezcla, las determinaciones obtenidas aparecen detalladas en el epígrafe resultados.

\section{Resultados}

\subsection{Curva granulométrica del Jabre}

Las características granulométricas de este material se han analizado en laboratorio mediante tamizado, siguiendo la norma UNE 103 101:95 (1995) con tamices UNE-EN 933-2 desprendiendo los valores que se expresan en la figura 1.

Con estos resultados obtenidos a partir de la curva granulométrica del material, no se podría estabilizar con cal este tipo de material en los suelos EST1 y EST2, puesto que no cumple el requisito de la tabla 512.1.a [12] que el porcentaje que pasa por el tamiz 0,063mm debe de ser superior o igual al $15 \%$ y en nuestro caso es significativamente inferior.

Para el caso de cumplir con la tabla 512.1.b de la misma orden ministerial, en este caso cumple todos los requisitos para poderse estabilizar con cemento, pudiendo ser S-EST1, S-EST2 y S-EST3.

Ahora bien, si nos vamos a la tabla 512.3.a, podemos descartar los S-EST1 y S-EST2 puesto que el índice plástico del jabre es muy bajo, no superando el $2 \%$.

Ya por último, observando la tabla 512.3.b sólo cabe clasificar este material como S-EST-3.

En este material existen fracciones de todos los tamaños comprendidos entre el más pequeño y el mayor del mismo, es decir, con una "granulometría continua".

De acuerdo con lo anteriormente expuesto, este material granular es óptimo para estabilizarse con el cemento y por esto se eligió el cemento como conglomerante. 


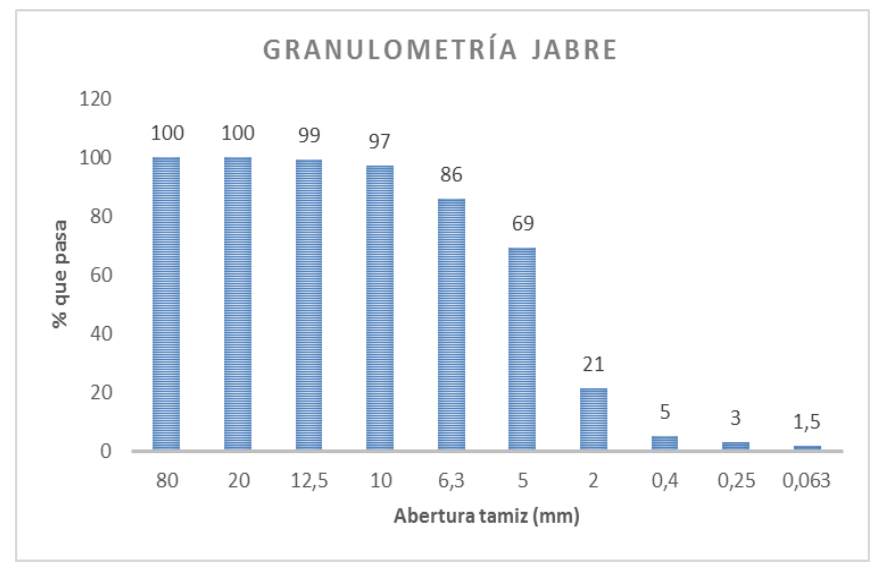

Figura 4: Curva granulométrica del jabre

\subsection{Plasticidad}

Para el límite líquido se utilizó el aparato de Casagrande. Se ensayó con diferentes humedades, siendo la más baja $20 \%$ y la más alta $27 \%$, sin embargo, hasta el rango de humedades comprendidas entre 21 y $24 \%$ ningún resultado daba un número de golpes comprendido entre 10 y 40 . Siendo el resultado del ensayo de 16 golpes para una humedad de $23 \%$ y de 35 golpes para una humedad del $21 \%$. Por lo tanto, el valor del límite líquido del jabre es de $22 \%$.

Los resultados del ensayo del Límite Plástico del jabre resultaron un valor de promedio de $24 \%$, por tanto, el Índice de Plasticidad (IP=LL-LP) es prácticamente nulo, asemejándose este material en cuanto a comportamiento a las arenas, debido a la muy poca proporción de finos. Esta es una propiedad muy positiva para el jabre, puesto que no presentará cambios significativos de plasticidad en presencia de agua, siendo suelos más estables y permeables.

\subsection{Ensayo de Compactación Proctor Normal}

Con el ensayo Proctor Normal se determinó la curva de humedad óptima-densidad máxima del jabre. En este ensayo se realizaron un total de 15 probetas compactándolas con unos porcentajes de agua a priori predefinidos, que, una vez realizado y visto su contenido de agua final, se calculó su densidad. Todos estos datos se llevaron al programa statgraphics y se obtuvo la parábola de regresión que mejor ajuste presentaba, de tal forma que una vez conocida ésta, se derivó su función y se sacó su máximo relativo, valor éste que relaciona su porcentaje de humedad óptima con su máxima densidad seca (figura 2).

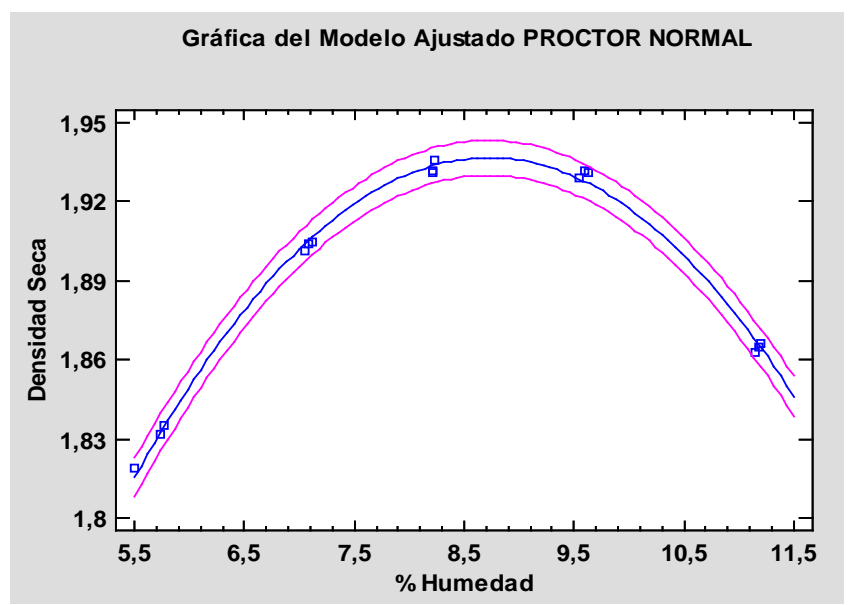

Figura 5. Modelo ajustado de Proctor Normal al jabre 


\section{CONGRESO IBÉRICO DE AGROINGENIERÍA \\ X CONGRESSO IBÉRICO DE AGROENGENHARIA \\ 3 - 6 septiembre 2019, Huesca - España}

Para el Proctor Normal, la parábola que explica la relación entre los datos observados y los predichos tiene una correlación $\mathrm{R}^{2}$ del $99,5 \%$. Al darnos un $\mathrm{p}$-valor de $0,000<0,05$, se puede inferir estadísticamente que la siguiente parábola explica la realidad con un nivel de confianza de al menos el 95\%:

\section{Dseca $=1,04783+0,203842 \cdot \mathrm{H}-0,0116878 \cdot \mathrm{H}^{2}$}

La máxima densidad obtenida fue de 1,93 kg/dm3 para una humedad óptima del 8,7\%.

Basándonos en los estudios de [13] y de [14], un adición de cemento induce a una ligera bajada de la densidad seca y a un aumento del agua óptima comparándolo con si realizamos el mismo proceso pero sin conglomerante. Esto es debido a la hidratación que debe de tener el cemento que le hemos añadido. Esto es importante tenerlo en cuenta ya que se incrementarían ligeramente los valores de resistencia al añadir a nuestro estudio ese porcentaje de agua de hidratación.

De la misma forma y tal y como nos indica [15] si se incrementase el contenido en arcilla de este material por sobre-descomposición del granito formándose caolín (silicato de aluminio hidratado) el contenido en cemento debe aumentarse, ya que como indica el mismo autor y lo corrobora [16] en su libro, obras en tierra, la adición de cemento reduciría el efecto no deseado de aumento de la plasticidad así como atenuar la expansión de la arcilla, consiguiendo con la adición de cemento, reducir el índice plástico de la mezcla, de tal forma que habría que añadir más cantidad de cemento, por cuanta más cantidad contenga de arcilla.

\subsection{Resistencia a compresión del jabre estabilizado con cemento}

\subsubsection{Análisis ANOVA de las muestras}

El jabre, una vez caracterizadas sus propiedades geotécnicas de granulometría, plasticidad y compactación, se mezcló con el cemento, y con el porcentaje de humedad óptimo que proporciona la máxima densidad, obtenido anteriormente (figura 3).

Tras realizar los ensayos de compresión (según UNE-EN 13286-41, 2003), se ha realizado un análisis multifactorial ANOVA que nos liga y discrimina la resistencia a compresión simple $(\mathrm{N} / \mathrm{cm} 2)$ con los porcentajes de cemento utilizados (0-1,5-2,5-3,5-4,5-5,5-6,5-7,5-8,5 y 10\%) a los distintos días a rotura ( 2,7 y 21 días) con 3 repeticiones por cada ensayo.

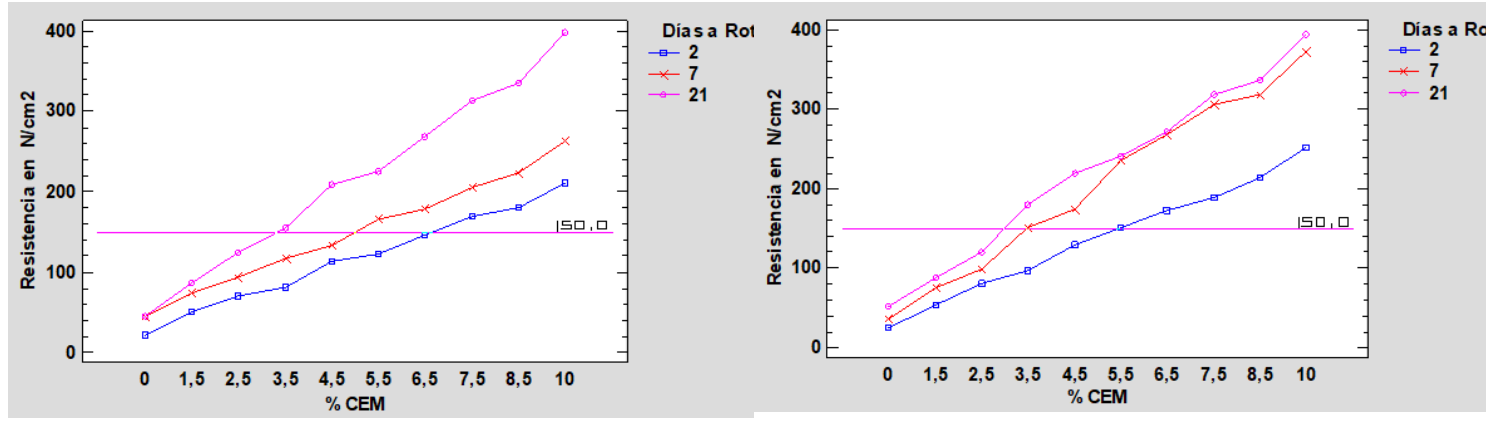

Figura 6. Resultados de la resistencia del jabre- CEM II B-L 32,5N

Figura 7. Resultados de la resistencia del jabre-CEM II A-V 42,5N

En la figura 3 se observan dos cosas básicamente: que las curvas a 2 y 7 días son prácticamente paralelas, y que la curva a 21 días de rotura tiene una pendiente mayor que las otras, alcanzando resistencias más altas a la misma concentración de cemento usada en la mezcla.

A simple vista en la figura 4 se observan dos cosas básicamente, la primera es que hay pocas diferencias entre la curva 7 y 21 días de rotura y la segunda, que la curva a dos días de rotura tiene, como cabía de esperar, menores resistencias. 


\section{CONGRESO IBÉRICO DE AGROINGENIERÍA \\ X CONGRESSO IBÉRICO DE AGROENGENHARIA \\ 3 - 6 septiembre 2019, Huesca - España}

En ambas figuras 3 y 4, la resistencia se ha tomado como referencia el valor de $150 \mathrm{~N} / \mathrm{cm} 2$, ya que el PG-3, así como su Orden FOM/2523/2014 (2014) nos define que la resistencia mínima a compresión simple en suelos estabilizados in situ ha de ser de 1,5 MPa $(150 \mathrm{~N} / \mathrm{cm} 2)$.

Se ha realizado un análisis estadístico ANOVA, introduciendo como variable dependiente la resistencia y como factores el \%CEM y días a rotura.

La tabla ANOVA descompone la variabilidad de Resistencia en contribuciones debidas a los dos factores (\%CEM y días a rotura). La contribución de cada factor se mide eliminando los efectos de los demás factores. Los valores-P prueban la significancia estadística de cada uno de los factores. Puesto que los 3 valores-P son menores que 0,05 , estos factores tienen un efecto estadísticamente significativo sobre Resistencia con un $95,0 \%$ de nivel de confianza, comprobándose que se cumple la homocedasticidad de los errores y que el ruido no es significativo.

A continuación se han analizado estas rectas de regresión, realizando un análisis estadístico de regresión lineal con análisis de la varianza. En todos los casos, la variable dependiente es la Resistencia ( $\mathrm{R}$, en $\mathrm{N} / \mathrm{cm} 2$ ) y la variable independiente el porcentaje de cemento (\%CEM).

\subsubsection{Análisis REGRESIÓN LINEAL de las muestras}

\section{Regresión Lineal para un curado de 2 días}

Variable dependiente: Resistencia y variable independiente: \%CEM

Jabre- CEM II B-L 32,5N

$\mathrm{R}=22,5849+19,1043 \cdot \% \mathrm{CEM}$
Jabre-CEM II A-V 42,5N

$\mathrm{R}=22,9746+22,7344 \% \mathrm{CEM}$

El estadístico R-Cuadrada indica que el modelo ajustado explica 99,7239\% de la variabilidad en Resistencia (N/cm2) para el caso del CEM II A-V 42,5N y del 99,0692\% para el CEM II B-L $32,5 \mathrm{~N}$. El coeficiente de correlación es superior al $99 \%$ en ambas muestras, indicando una relación relativamente fuerte entre las variables.

\section{Comparación de ambas muestras:}

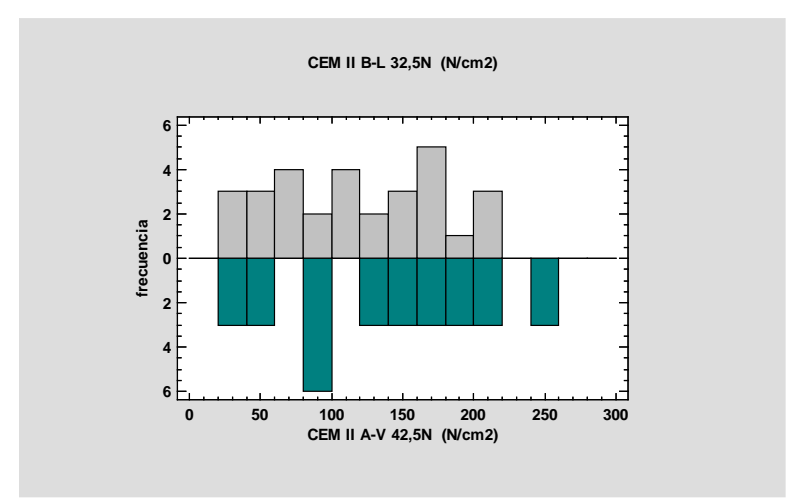

Figura 8. Histograma para el curado 2 días.

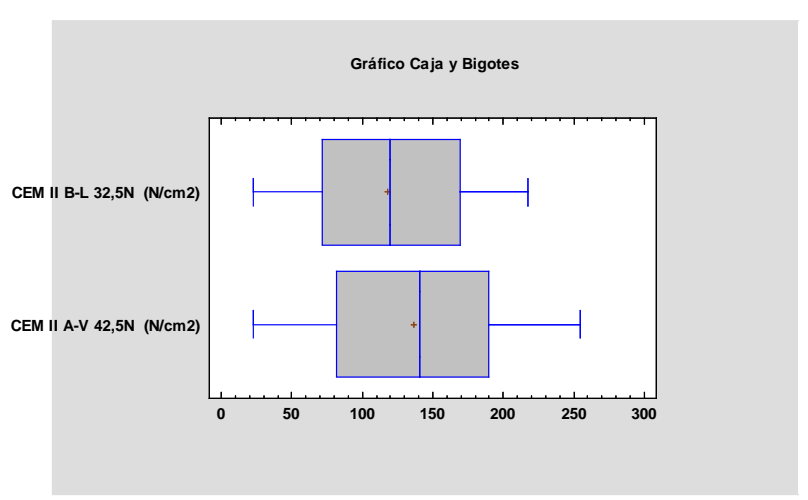

Figura 9. Gráfico de caja y bigotes para el curado 2 días

Realizando un estudio estadístico comparativo de ambas muestras de Prueba $t$ para comparar medias

$$
\begin{aligned}
& \text { Hipótesis nula: media1 = suponiendo varianzas iguales: } \\
& \text { media2 } \\
& \text { Hipótesis Alt.: media1 <> } \\
& \text { media2 } \\
& \mathrm{t}=-1,10542 \text { valor }-\mathrm{P}=0,27354
\end{aligned}
$$

No se rechaza la hipótesis nula para un nivel de significación de 5\%. 


\section{CONGRESO IBÉRICO DE AGROINGENIERÍA \\ X CONGRESSO IBÉRICO DE AGROENGENHARIA \\ 3 - 6 septiembre 2019, Huesca - España}

En este caso, la prueba se ha construido para determinar si las medias son iguales versus la hipótesis alternativa de que son diferentes. Puesto que el valor-P calculado es mayor del $27 \%$ podemos afirmar con un $5 \%$ de significancia que no se puede rechazar la hipótesis nula, resultando que las medias son parecidas o mejor dicho, no son distintas. No hay diferencia significativa entre las medias de las dos muestras de datos, con un nivel de confianza del 95,0\%.

Realizando el estudio estadístico Prueba-F comparativo de ambas muestras para comparar desviaciones típicas.

$$
\begin{aligned}
& \text { Hipótesis Nula: sigma1 }=\text { sigma2 } \\
& \text { Hipótesis Alt.: sigma1 }<\text { sigma2 }
\end{aligned}
$$

No se rechaza la hipótesis nula para un nivel de significación de $5 \%$.

En este caso, la prueba se ha construido para determinar si las desviaciones típicas son iguales versus la hipótesis alternativa de que son diferentes. Puesto que el valor-P calculado es mayor del $36 \%$ podemos afirmar con un $5 \%$ de significanciaq que no se puede rechazar la hipótesis nula, resultando que las desviaciones típicas son parecidas o mejor dicho, tenemos que aceptar que las dispersiones son iguales.

\section{$\underline{\text { Regresión Lineal para un curado de } 7 \text { días }}$}

Variable dependiente: Resistencia y variable independiente: \%CEM

$$
\begin{array}{cc}
\text { Jabre-CEM II A-V 42,5N } & \text { Jabre- CEM II B-L 32,5N } \\
\mathrm{R}=26,3428+35,4181 \cdot \% \text { CEM } & \mathrm{R}=40,3604+22,2239 \cdot \% \text { CEM }
\end{array}
$$

El estadístico R-Cuadrada indica que el modelo ajustado explica 98,9591\% de la variabilidad en Resistencia (N/cm2) para el caso del CEM II A-V 42,5N y del 99,3509\% para el CEM II B-L $32,5 \mathrm{~N}$. El coeficiente de correlación está entorno al 99\% en ambas muestras, indicando una relación relativamente fuerte entre las variables.

\section{Comparación de ambas muestras:}

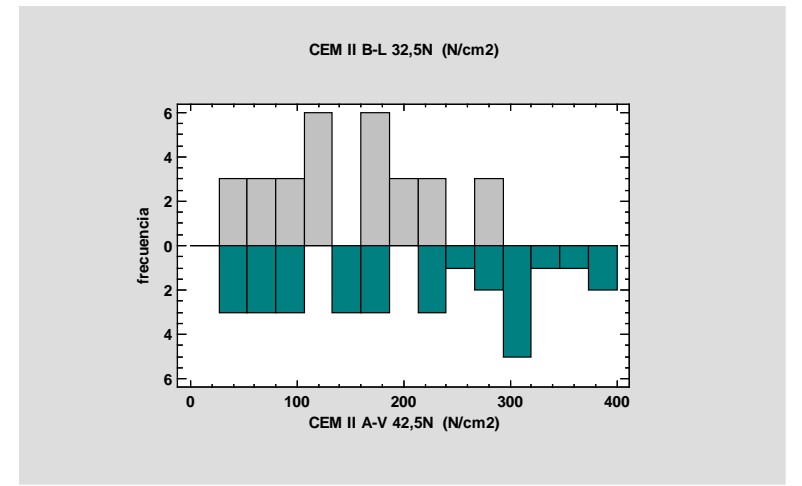

Figura 10. Histograma para el curado 7 días.

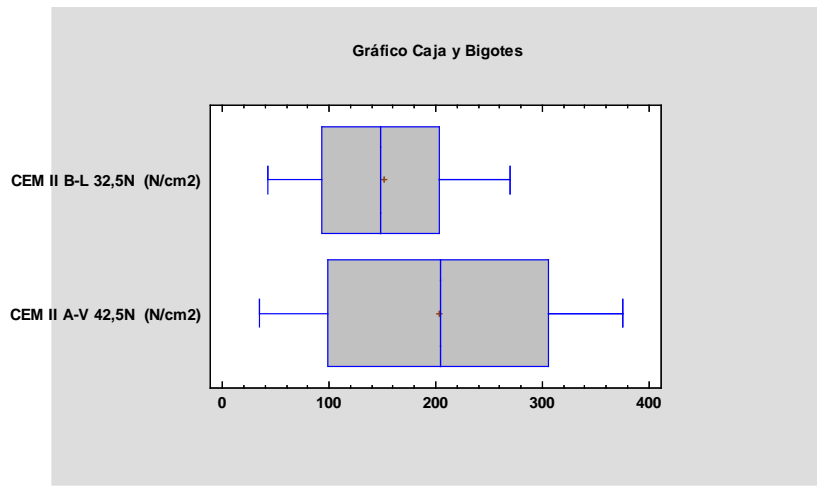

Figura 11. Gráfico de caja y bigotes para el curado 7 días

Realizando un estudio estadístico comparativo de ambas muestras de Prueba t para comparar medias. media2

Hipótesis nula: media1 = suponiendo varianzas iguales:

media2

Hipótesis Alt.: media1 <>

Se rechaza la hipótesis nula para un nivel de significación de 5\%.

En este caso, la prueba se ha construido para determinar si las medias son iguales versus la hipótesis alternativa de que son diferentes. Puesto que el valor-P calculado es del 3,2\%, y por lo 


\section{CONGRESO IBÉRICO DE AGROINGENIERÍA \\ X CONGRESSO IBÉRICO DE AGROENGENHARIA \\ 3 - 6 septiembre 2019, Huesca - España}

tanto inferior al 5\%, podemos afirmar con un 5\% de significancia que sí se puede rechazar la hipótesis nula, aceptando la alternativa, resultando que las medias son distintas.

Realizando el estudio estadístico Prueba-F comparativo de ambas muestras para comparar desviaciones típicas.

$$
\begin{aligned}
& \text { Hipótesis Nula: sigma1 }=\text { sigma2 } \\
& \text { Hipótesis Alt.: sigma1 }>\text { sigma2 }
\end{aligned}
$$$$
\mathrm{F}=0,39217 \text { valor- } \mathrm{P}=0,014062
$$

Se rechaza la hipótesis nula para un nivel de significación de $5 \%$.

En este caso, la prueba se ha construido para determinar si las desviaciones típicas son iguales versus la hipótesis alternativa de que son diferentes. Puesto que el valor-P calculado es del $1,4 \%$, y por lo tanto inferior al $5 \%$, podemos afirmar con un $5 \%$ de significancia que sí se puede rechazar la hipótesis nula, resultando que las medias son distintas y por tanto, las dispersiones no son iguales.

Regresión Lineal para un curado de 21 días

Variable dependiente: Resistencia y variable independiente: \%CEM

Jabre-CEM II A-V 42,5N

$\mathrm{R}=46,9618+34,985 \%$ CEM
Jabre- CEM II B-L 32,5N

$\mathrm{R}=39,8316+35,5063 \cdot \% \mathrm{CEM}$

El estadístico R-Cuadrada indica que el modelo ajustado explica 99,1698\% de la variabilidad en Resistencia (N/cm2) para el caso del CEM II A-V 42,5N y del 99,5534\% para el CEM II B-L $32,5 \mathrm{~N}$. El coeficiente de correlación es superior al $99 \%$ en ambas muestras, indicando una relación relativamente fuerte entre las variables.

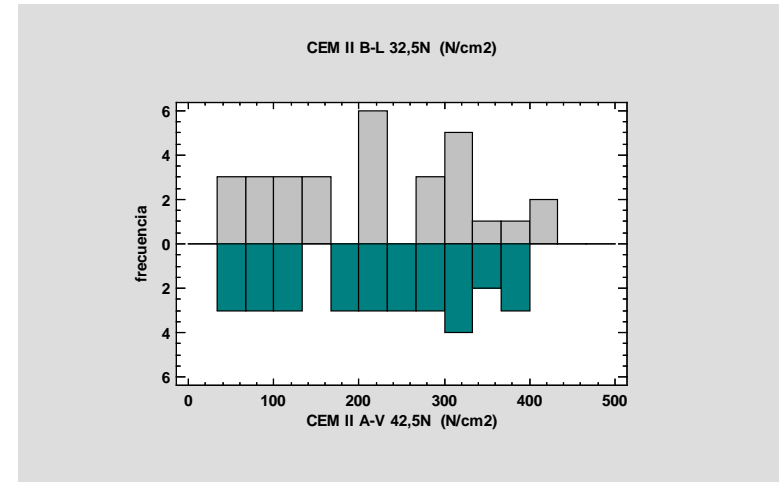

Figura 12. Histograma para el curado 21 días.

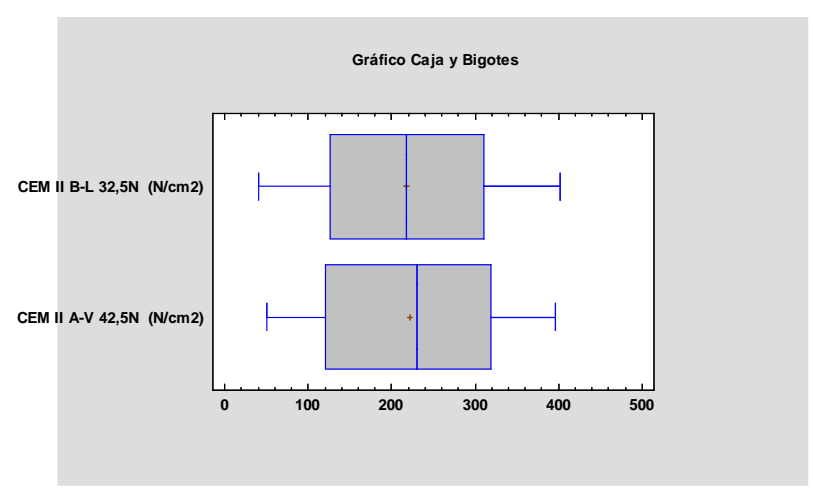

Figura 13. Gráfico de caja y bigotes para el curado 21 días

Realizando un estudio estadístico comparativo de ambas muestras de Prueba $t$ para comparar medias

Hipótesis nula: media1 $=$ media2

Hipótesis Alt.: media1 $<$ media2

suponiendo varianzas iguales:

$\mathrm{t}=-0,1606$ valor- $\mathrm{P}=0,872966$

No se rechaza la hipótesis nula para un nivel de significación de $5 \%$.

En este caso, la prueba se ha construido para determinar si las medias son iguales versus la hipótesis alternativa de que son diferentes. Puesto que el valor-P calculado es mayor del $87 \%$ podemos afirmar a un $5 \%$ de significancia que no se puede rechazar la hipótesis nula, resultando que las medias son parecidas o mejor dicho, no son distintas. No hay diferencia significativa entre las medias de las dos muestras de datos, con un nivel de confianza del 95,0\%.

Realizando el estudio estadístico Prueba-F comparativo de ambas muestras para comparar desviaciones típicas.

Hipótesis Nula: sigma1 = sigma2

$$
\mathrm{F}=1,02606 \text { valor }-\mathrm{P}=0,945251
$$

Hipótesis Alt.: sigma1 $<$ sigma2

No se rechaza la hipótesis nula para un nivel de significación de 5\%. 


\section{CONGRESO IBÉRICO DE AGROINGENIERÍA \\ X CONGRESSO IBÉRICO DE AGROENGENHARIA \\ 3 - 6 septiembre 2019, Huesca - España}

En este caso, la prueba se ha construido para determinar si las desviaciones típicas son iguales versus la hipótesis alternativa de que son diferentes. Puesto que el valor-P calculado es mayor del $94 \%$ podemos afirmar a un 5\% de significancia que no se puede rechazar la hipótesis nula, resultando que las desviaciones típicas son parecidas o mejor dicho, tenemos que aceptar que las dispersiones son iguales.

\subsubsection{Resistencia a compresión y normativa.}

De acuerdo con el mínimo requerido de resistencia en los suelos estabilizados insitu, según el PG-3 así como su Orden [17] que nos define que esa resistencia mínima a compresión simple ha de ser de 1,5 MPa $(150 \mathrm{~N} / \mathrm{cm} 2)$, y considerando el modelo estadístico con sus rectas de regresión e igualando este valor sobre las rectas de regresión, se determina que aplicando un Proctor Normal del 100\% a una humedad del 8,7\% (valor óptimo humedad/densidad), se alcanza una resistencia mínima de 1,5 MPa con una concentración mínima en función de cada tipo de cemento de:

Para el CEM II B-L 32,5N se necesitaría un mínimo de concentración de cemento del 3,5\% dejando un tiempo de fraguado de 21 días, aunque si se quiere disminuir el tiempo, la concentración de cemento aumenta hasta el 5\%.

Para el CEM II A-V 42,5N con este valor mínimo del 3,5\% de cemento se alcanza de manera muy similar esta resistencia de referencia tanto a los 21 días de fraguado como a los 7. En parte, este comportamiento se puede explicar debido a que este cemento tiene más resistencia y por lo tanto se alcanzaría esa resistencia mínima antes en comparación con el anterior cemento.

Conclusión parecida es a la que llega[18]. en el caso de una estabilización de un suelo de filitas estabilizado con cemento V-A 32,5 N/mm2, indicando que es conveniente incorporar un $5 \%$ de cemento para mejorar la resistencia a punzonamiento del suelo y por ende la resistencia de este, registrándose con esta proporción un incremento de la resistencia a compresión uniaxial y reduciéndose la permeabilidad y así poder con los requisitos mínimos del PG-3. También, en este estudio se descarta la estabilización con cal, por aumentar la permeabilidad.

\section{Conclusiones}

Se concluye que el jabre es un material granular con unas buenas propiedades de granulometría, plasticidad y compactación. Mezclado con cemento CEM II B-L 32,5N, se ha comprobado la influencia significativa del \% de cemento en la mezcla y del tiempo de curado.

Del estudio estadístico comparativo de medias y desviaciones típicas se desprende, a tenor de su p-Valor, que la que más correlación tiene entre sus muestras es la serie de rotura a 21 días, siendo sus rectas de regresión muy similares. A pesar de ser cementos con resistencias distintas, la conclusión que se puede extractar es que se puede utilizar un cemento más económico como es el CEM II B-L 32,5N, obteniéndose resultados similares en cuanto a resistencia se refiere al dejar pasar 21 días de fraguado.

La última conclusión, casi es más una recomendación de carácter práctico, puesto que la resistencia mínima a compresión simple, según PG-3, debe de ser de 1,5 $\mathrm{MPa}(150 \mathrm{~N} / \mathrm{cm} 2)$, valores estos que se alcanzan en función de cada tipo de cemento con:

CEM II B-L 32,5N

CEM II A-V 42,5N
3,5\% a los 21 días, y del 5\% de cemento a los 7 días.

Valores inferiores al 5\% de cemento para estos tiempos de curado harían inviable la obra por falta de resistencia.

$3,5 \%$ a los 7 y 21 días.

Valores inferiores del 3,5\% de CEM harían inviable la obra por falta de resistencia. 


\section{CONGRESO IBÉRICO DE AGROINGENIERÍA \\ X CONGRESSO IBÉRICO DE AGROENGENHARIA \\ 3- 6 septiembre 2019, Huesca - España}

\section{Referencias}

1. Gallo, I.M., Vías romanas: ingeniería y técnica constructiva2004: Isaac Moreno Gallo.

2. Mejora en las aceras con arena de jabre, 2016: La Moraleja, Entidad urbanística de conservación.

3. RC-16, Instrucción para la recepción de cementos. Real Decreto 256/2016, de 10 de junio. Ministerio de la Presidencia, 2016.

4. Calleja, J., Cenizas, cementos y hormigones con cenizas. ETCC/CSIC Madrid, 1982.

5. Montes, J.G., Identificación de una carretera romana a través de la procedencia de los materiales. Elementos de Ingeniería Romana.Congreso Europeo Obras Públicas Romanas, 2004.

6. Pretel, G.B., PG-3 Pliego de prescripciones técnicas generales para obras de carreteras y puentes. Artículo 513, 2010: Ediciones Liteam SL.

7. UNE103101:95, Análisis granulométrico de suelo por tamizado. AENOR, 1995.

8. UNE103103:93, Determinación del Límite Líquido de un suelo. AENOR, 1993.

9. UNE103104:93, Determinación del Límite Líquido de un suelo. AENOR, 1993.

10. UNE103500:94, Geotécnia. Ensayo de compactación. Proctor Normal. AENOR, 1994.

11. UNE-EN13286-41, Mezclas de áridos sin ligante y con conglomerante hidráulico. Parte 41: Método de ensayo para la determinación de la resistencia a la compresión de las mezclas de áridos con conglomerante hidráulico. AENOR, 2003.

12. FOM/2523/2014, O.M., Artículo 512. Suelos estabilizados in-situ. 2014.

13. Kezdy, A., Stabilized earth road. Scientific Pub. Co, Amsterdam, 1979.

14. Miller, G.A., Azad, S., Influence of soil type on stabilization with cement kiln dust. Construction adn Building Materials 14,89-97, 2000.

15. Dal-Ré, R., Caminos rurales. Proyecto y construcción. Ediciones Mundi-Prensa, 1994.

16. Cuadra J., G.C., Obras en tierra. Universidad Politécnica de Madrid. Escuela Universitaria deIngeniería Técnica Agrícola, 2003.

17. FOM/2523/2014, B.d.e.d.O., Orden por la que se actualizan determinados artículos del pliego de prescripciones técnicas generales para obras de carreteras y puentes, relativos a materiales básicos, a firmes y pavimentos, y a señalización, balizamiento y sistemas de contención de vehículos., in Artículo 512.3 tipo y composición del sulo estabilizado2014.

18. Garzón E., C.M.e.a., Effect of cement and lime on phyllites raw materials from SE Spain. 17th International Congress on Proyect Management and Engineering, 2013. Universidad de Almería e Instituto de Ciencia de Materiales, CSIC-US. 